\title{
Reference frame linking and tests of GR with Gaia astrometry of asteroids
}

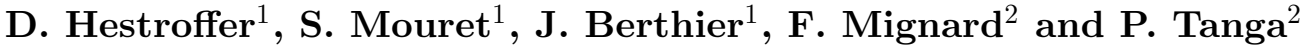 \\ ${ }^{1}$ IMCCE, Observatoire de Paris, CNRS, \\ 77 av. Denfert-Rochereau, F-75014, Paris, France \\ email: hestro@imcce.fr \\ ${ }^{2}$ Laboratoire Cassiopée, observatoire de la Cote d'Azur, CNRS \\ Mont Gros, F-06000, Nice, france \\ email: mignard@oca-nice.fr
}

\begin{abstract}
The Gaia satellite, an ESA cornerstone mission to be launched at the end of the year 2011, will observe a large number of celestial bodies including also small bodies of the solar system. Albeit spread from the inner to the outer regions of the solar system, these are mainly near-Earth objects and main-belt asteroids. All objects brighter than magnitude $V \leqslant 20$ that cross the field of view (i.e. with solar elongation $45^{\circ} \leqslant L \leqslant 135^{\circ}$ ) of the survey-mode scanning telescope will be observed. The mission will provide, over its 5 years duration, high precision photometry and astrometry with an unprecedented accuracy ranging roughly from 0.3 to 3 milli-arcsecond on the CCD level, and depending on the target's magnitude. In addition, several hundreds of QSOs directly observed by Gaia will provide the kinematically non-rotating reference frame in the visible light, resulting in the construction of a 'Gaia-ICRF'.

The positions of the asteroids hence enable to relate the dynamical reference frame - as defined by the equations of motion - to the kinematic one, and to further check the non-rotating consistency between both frames' definition. Here we show the results of a variance analysis obtained from a realistic simulation of observations for such a link. The simulation takes into account the time sequences and geometry of the observations that are particular to Gaia observations of solar system objects, as well as the instrument sensitivity and photon noise. Additionally, we show the achievable precision for the determination of a possible time variation of the gravitational constant $\dot{G} / G$. Taking into account the non-completeness of the actually known population of NEOs, we also give updated values for the nominal precision of the joint determination of the solar quadrupole $J_{2}$ and PPN parameter $\beta$.
\end{abstract}

Keywords. asteroids, space vehicles, astrometry, reference systems

\section{Introduction}

Ten years have passed now since the publication of the Hipparcos catalogue. The next generation of space astrometry mission Gaia will overpass the already important results achieved or enabled by the Hipparcos/Tycho astrometric catalogues. In addition to the observations of the stars in our Milky-Way, Gaia will also detect and observe a large number of 'secondary' sources: the distant quasars, and the nearby solar system objects. Indeed, Gaia will observe almost all celestial bodies brighter than $V \leqslant 20$, hence $\approx 300,000$ asteroids; mainly near-Earth objects (NEOs) and main-belt asteroids (MBAs). Among the many scientific outputs from Gaia observations of asteroids (see e.g. Mignard et al. 2007) the highly accurate astrometry of asteroids will enable dramatic orbit improvements, and also detection of such a subtle effect as the orbit perturbations. These concern the positioning of the equinox and ecliptic in the optical-ICRS, and the test of a possible net rotation between the two supposedly non-rotating frames. It also concerns a test of GR from relativistic effects, and variation of the constant of gravity. 


\section{Results}

All observed directions are related to the Gaia reference frame which at the end is itself materialized by primary sources and the QSOs. The computed positions on the other hand are given by the equations of motion of the target and the Gaia platform, all connected to the dynamical reference frame materialized by the ecliptic and equinox. By definition both frames are non-rotating. Differences between the observed and computed directions can arise from errors in the dynamical model (initial, conditions, masses, orbit of the Earth, etc.) and/or from the fact that they are not expressed in the same frame. One possible parameterization is to introduce a rotation $\Omega$ and rotation rate $\dot{\Omega}$ between the two frames. Similarly a temporal change of the constant of gravity $G$ will perturb all orbits. Simulation over 180,000 retained objects yields a formal precision of $\sigma_{\Omega} \sim 15 \mu$ as and $\sigma_{\dot{\Omega}} \sim 6 \mu$ as/year, corresponding to testing a possible rotation rate at a $3 \times 10^{-11} \mathrm{rad} /$ year level.

The major effect of GR on the orbit is a precession of the perihelion. In the parameterized post-Newtonian (PPN) formalism, this effect depends on the parameter $\beta$ when $\gamma$ is supposed to be known with enough accuracy by other means. Taking into account their eccentricities, it appears that highly eccentric NEOs are as much sensitive to this effect than Mercury is. However not all NEOs are currently known and more are to be discovered. Samples of hypothetical populations can then be created following the un-biased orbital and size distribution given by Bottke et al. (2002). It turns out that more highly eccentric orbits are likely to be found prior to Gaia launch. Another effect coupled with the relativistic one, arises from the unknown solar quadrupole $J_{2}$, since both will provide secular drift of the perihelion. Nevertheless this effect can, in theory, be decoupled from the relativistic one by observing a large number of bodies well distributed in the $(a, e)$ plane. We have generated three different samples of possible NEOs and computed either separately or simultaneously the PPN parameter $\beta$ and the solar $J_{2}$. As a result $\beta$ and $J_{2}$ can be derived simultaneously with a precision of $\sigma_{\beta} \sim 5 \times 10^{-4}$ and $\sigma_{J_{2}} \sim 1.5 \times 10^{-8}$. Since the correlation is not too high (corresponding to the case where both parameters cannot be obtained simultaneously), these results will be slightly improved when one considers only one of the parameters in the fitting procedure.

\section{Discussion}

These results are encouraging and will provide complementary information to those obtained by other techniques (Lunar Laser Ranging, planetary ephemerides, helioseismology) and involving other models. While the catalogue of known MBAs is almost complete down to magnitude 20, more NEOs and inner-Earth orbits are still to be discovered with current surveys and Gaia. One should note also that systematic effects due to non-modeled non-gravitational effect could degrade these results. Further, similar studies could be applied to test the SEP through a measure of Nordtvedt parameter $\eta$, and post-Einsteinian metric theories of relativity (Jaekel \& Reynaud 2006), by involving the motions of Trojans, dormant comets and Centaurs in particular.

\section{References}

Mignard, F., Cellino, A., Muinonen, K., Tanga, P., Delbò, M., Dell'Oro, A., Granvik, M., Hestroffer, D., Mouret, S., Thuillot, W., \& Virtanen, J. 2007, Earth, Moon, and Planets, 101, 97-125.

Bottke, W. F., Morbidelli, A., Jedicke, R., Petit, J.-M., Levison, H. F., Michel, P., \& Metcalfe, T. S. Icarus, 156, 399.

Jaekel, L. \& Reynaud, S. 2006, Class. Quantum Grav., 23, 777-798. 